Das Bedurfnis, Goethes Faust in der Gestalt, in welcher der Dichter sein Werk zuerst der Öffentlichkeit vorlegte, in Händen zu baben, machte sich stärker fühlbar, seit Scherers Untersuchungen für die Entstehungsgeschichte der einzelnen Teile der Dichtung neue Bahn gebrochen haben. Das gleiche Ziel wie Scherer in Goethes Frühzeit (Quellen und Forschungen XXXIV. Strassburg 1879) verfolgen die Ausführungen Schröers in der Einleitung zu seiner Ausgabe des ersten Faustteiles (Heilbronn 1881). Die Mitteilung und Prüfung der Ergebnisse hat hier ebenso wenig Raum wie die Aufzählung aller Litteratur, welche diese Frage der Faustforschung berührt. Dienlicher mag der Hinweis auf neue Stützpunkte zur Lösung der schwierigen Aufgabe sein.

Im Augusthefte des 'Teutschen Merkur' 1773 (III 133 ff.) erschien Wielands lyrisches Drama 'Die Wahl des Herkules.' Es ist sein Faust, nur dass statt des Durstes nach Wahrheit der Eifer für Tugend den Kern bildet; ein Unterschied, der für den Fortschritt der geistigen Entwickelung des 18. Jahrhunderts bezeichnend ist und Wielands Abstand von Goethe zum Ausdruck bringt. Abgesehen von dieser Grundverschiedenheit der Auffassung aber sind beide Helden himmelstürmende Titanen. Herkules zerbricht die Fesseln Amors, Faust wirft die Bande der Fachgelahrtheit ab. Gottähnlichkeit, Göttlichkeit streben beide an. Darin sind 
sie Brtder. Und diese Verwandtschaft war es, welche Wielands Herkules mit Goethes Faust verknipft hat.

Wielands Held ruft ans: 'Ein feiger Sklave sollt' ich sein? Beim Himmel, nein! Ich fahl ein Herz in meinem Busen schlagen!.. 0 0 Götter, darf ichs wagen, ... Um ein Gehoimnis ench zn fragen? .... Diese Glut in meinem Busen, .. Dieses Hupfen jeder Ader, wo andre beben, ... Wie nenn' ichs, was den andern Erdensöhnen mich So ungleich macht? .. Was auf den ganzen Kreis von ihren kleinen Sorgen, Entwtrfen, Freuden, Plagen, Kalt und unbewegt mich niederblicken heisst?' (3. 133 f.) So verwthscht auch Faust seine Vergangenheit und sucht durch Geistesmund manch Geheimnis zu erkanden; auch er fuhlt junges Lebensgluck Neugl th hend sich durch Nerv' und Adern rinnen; anch er weiss sich erhaben uber andere Menschen und kennt nicht ihre Furcht (V. 23 ff. 79 f. 13 ff.). Herknles stellt die Frage: ' $W$ er bin ich? Gab ein Halbgott, Gab ein Gott das Leben mir? Wie wallt mein Blut Bei diesem grossen Gedanken anf! Ich zittre nicht Indem ich ihn zu denken wage!.. I ch fuhl's, ich fuhl's, Es ist der Götter Blut, was diese Adern schwellt! 0 du, der mir das Leben gab, Unsterblicher, warum verbirgst du dich vor mir? 0 zeige dich!' (S. 134 f.) Ähnlich lässt sich Faust zu einer Go t t heit auf a c b w ell en (V.140.1958) und fragt : 'Bin ich ein Gott? Mir wird so licht.. Schon ' $\|_{h} l^{\prime}$ ' ich meine Kräfte höher.. Ich fuhle Muth mich in die Welt zu wagen.. I c h fabl's, da schwebst am mich, erflehter Geist! Enthulle dich!' (V. 86. 109. 111. 122 f.) Den Einwa gegen diese U'berschatzung, welchen in Goethes Faust der Geist erhebt, macht sich Herkules selbst: 'Aber ... wenn die selbst.betrogne Vermessne Seele, was sie feurig wunscht, Für A hnung hielte? Alcid, du trüumst, du träumst von Gottheit? du?' (S. 135) wie Faust von seiner Kraft spricht, die ahn angsvoll sich vermass und empfin- 
det, dass er Gott nicht gleicht (S̈chröer V. 268. 299). Und ruft Herkules aus: 'Wie gross! wie klein! Izt, mathig, jedem Lngebeuer Trotz za bieten, Izt, verzagt vor einem Blicke! Izt, ganz durchdrungen von der hohen Schönheit Der Tugend, ganz von ihrer G ottheit voll,... Doch bald .... So niedrig sollt ich sein? So schwach! . . Eurer Le h re n so uneingedenk, Ihr Fürer meiner Jugend!' (S. 137) so äussert sich Faust: 'Ich, Ebenbild der Gottheit, das sich schon ganz nah gedunkt dem Spiegel ewger Wabrheit... Ein Donnerwort hat mich hinweggerafft .. So Latt' ich dich zu balten keine Kraft .. Ich füblte mich so klein, 80 gros B ... Wer lehret mich?' (Schröer V. 261 f. 269. 272. 274. 277) Ferner, wie die Wollust dem Herknles zuruft: 'Du fliehst die Welt, Alcid?. . E Entweichst da ihr in einen oden Wald; Sprichst mit dir selber, staunst, verlierst dich in Gedanken, $Z_{\text {weifelst, welchen Weg des }}$ Lebens Du nehmen sollst? Sieh eine Freundin, Die willig ist, zum Glück der Götter dir Len Weg zu zeigen !' (S.138 f.) so sucht Mephistoplueles Faust in W ald nnd Höhle auf, so spricht er: 'In die Welt weit Ans der Einsamkeit.. Wollen sie [die Geister] dich locken. Hör auf mit deinem Gram zu spielen ... willst du mit mir vereint Deine Schritte durchs Leben nehmen, So will ich mich geru bequemen Dein zı sein. ' (Schroer V. 1278 ff.) Wie Faust den Mephistopheles nach seinem Namen fragt (Schröer V. 974) so allch Herkules die Wollust (S. 139). Mit Genuss will sie Herkules gewinnen (S. 140) wie Mephistopheles den Faust (Schrðer V. 1337 fi.). Als letzten Trumpf, den sie gegen dio Tugend ansspielt, verspricht sie Herkules seine geliebte Dejanira (8. 148); und Mephistopheles lockt Fanst ans der Waldhoble durch Gretchen (V. $1976 \mathrm{ff}$.). Wie Herkules zwischen dem Guten and Bösen schwankend ansruft: 'Zw00 Seelen - $\mathbf{Z n}$ gewiss fábl ichs! - Z $\mathbf{W} 00$ Soelon kumpfen in meiner Brust!' (S. 147) so klagt Fanst: 'Zwe i Seelen wohnen, ach! in meiner 
Brast !' (Bchrōer V. 759)*). Ferner, wer wird durch Herknles' Arie: 'O trag Erbarmen Mit meinem Schmerz! Der innre Aufruhr Zerreisat mein Herz.... Glich meinem Sehmerzen Wohl je ein Bchmerz?' (S. 149 f.) nicht erinnert an das so viel herrlichere Gebet Gretchens im Zwinger? (V. 2047 fi. vgl. Goethe-Jahrbuch I $187 \mathrm{ff}$.) Noch manche einzelne Stelle lasst sich zur Vergleichnng heranziehen. So sagt die Tugend: 'Allein des wahren Gluckes Q a ell e Liegt in deiner e ig n e n Brust' (S. 152) und Faust: 'Erquickung hast du nicht gewonnen, Wenn sie dir nicht ans eigner Seele quillt' (V. 215 f.). Auch das freie Versmass, die ungleich wechselnden langen und kurzen Verszeilen des lyrischen Dramas waren fur den Fanst formgebend, wie umgekehrt ein Blick anf die Utberarbeitnng des Singspieles in der Ausgabe letater Hand (Bd. 26) lebrt, dass Wieland seine Verse nacb dem Vorbilde des Faust ordnet, wobei er sogar da und dort den Ausdruck nach Goethes Worten modelt; so liest man im 'Merkur' (S. 143): 'Die Zeit ist kostbar! Willst du sie verlieren?' in den Werken aber steht $(26,169)$ mit Racksicht auf Fanst (V. 205 f. 266) : 'Die Zeit ist kostbar, kurz das Leben.'

Kanm wird ein nubefangener Leser anf diese $\mathrm{Za}$ sammenstellangen antworten mit Wielands Versen: Wir lassen dies alles gern den Leuten, die auf entdeckte Ähnlichkeiten sich viel $\mathrm{zu}$ gute thun. Und wer ihre Berechtigung zogesteht, wird auch die Schlulsse daraus ziehen. Vor allem wird man die verglichenen Verse des Fanst nicht vor den August 1773 setzen durfen, freilich auch nicht behaupten können, dass sie kurz darnach entstanden sein müssen, da nicht nar die erste Lekture

*) Zugleich sei hier die Quelle dieses Ansdruckes anfgedeckt: Wieland merkt im Neuen Amadis (1771 II 15) an, Araspes in Xenophons Cyropädie finde zwei Seelen in seinem Herzen. Die Stelle im 6. Bnch, Kap. 1 \& 41 lautet: Súo

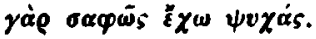


der Wielandischen Lichtung, sondern ebenso jede spatere den Einfluss geulbt haben kann. Las erstere ist an sich und anch darum wahrscheinlicher, weil fest steht, dass Goethe 1773 am Faust thätig war. Damit wird eine der Stellen (V. 122 f.l, welche nach Scherers Ansicht (Q F. XXXIV 82) aus Prosa umgeschrieben iat, spater angesetzt : sie ist reimlos und freirhythmisch wie Wielands Singspiel. Aber die von Scherer erkannto Zusammengehörigkeit der Scenen : Monolog mit Erdgeist, Fanst und Mephistopheles, Zwinger (Q. F. XXXIV 98 f.) wird durch diese Beobachtungen bestätigt; das von jenem mit den Worten: Mangel an Lokalfarbe, rein lyrisch, seelenmalende Monologe bezeichnete Gepräge dieser Sttucke ist nun durch das gleichgeeigenschaftete Vorbild erklart. Ferner ergibt sich, was schröer aus andern Grunden za erweisen sncht, dass zar 'grossen Lucke' schon in dieser Zeit Ansatze vorhanden waren.

Wer die Vergleichung im ganzen ins Ange fasst, dem wird die Vermutung nicht allzu gewagt erscheinen, dass anf einer Stufe der Entwurf des Faust dem Herkulesdrama ähnelte. Herkules, verzweifelt an seiner Vergangenheit, will in die Einsamkeit sich zurtickziehen; ebenso geht Fanst in die Einsamkeit (vgl. Scherer Q. F. XXXIV $84 \mathrm{f}$. Schröer V. 1279, eine Entwicklung, die also nioht dem prosaischen Faust schon angehört haben musste. Da tritt ihnen die Verfuhrung entgegen als Wollust und Mephistopheles. Die allegorische Erscheinung der auf ein Buhebette hingegos senen Wollust, von Herknles mit dem Ausrufe: 'Welch oln Anblick !. . trum ich wachend!' (S. 138) begleitet, konnte Vorbild gewesen sein fir die Spiegelscene, welcho Fanst mit den Worten schildert: 'Welch ein himmliseh Bild.. Muss ich in diesem hingestreckten Leibe Den Inbegriff von allen Himmeln sehn?.. Ich werde schier verrtckt ...' (V. 892 ff. 919). Dann ware diese in Italien fertig gestellte Scene Herenktuche alteren Ursprungs, wie auch Scherer vermutet hat (Q.F. XXXIV 
107). Der Hnuptteil des Wielandischen Singspieles, die Reden der Tugend, dichterisch dem Anfang weit nachstehend, thbte keinen greifbaren Einfluss anf Fanst. Der Schluss jedoch zielt anf die im zweiten Teile des Fanst gegebene Losung: schaffendes Wirken für die Menschheit (S. 143, 153) als Lebensanfgabe und als Preis die Aufnahme in den Himmel (S. 153).*)

Nicht nur die Wahl des Herkules, noch sndere Dichtungen Wielands wirkten, wenn anch entfernter, im Fanst nach. Auch an der Musarion, von der Goethe jedes Blatt auswendig gelernt hatte (Goethe-Jahrbuch II 381), ist seine Ausdrucksweise emporgewachsen. Schroer hat fur die Verse (Schröer 299 f.) im Faust : 'Den Góttern gleich ich nicht!... Dem Wurme gleich ich' an ahnliche Stellen in Goethes Epistel an Riese erinnert. Naher zu liegen scheint mir die Parallele mit den Worten in der Musarion (1768 S. 23): 'Mich bald zum Gott und bald zum Wurm zu machen.' Ebenso lusst vielleicht dio Vergleichung folgender Stellen eine dunkle Reminiscenz erkennen. In der Masarion wird ein 'Titans Söhnen gleich die Geisterwelt erstürmender' Mensch also geschildert (S. $8 \mathrm{f}$.) : 'Wie prächtig klingt's, den fesselfreien Geist Im reinen Quell des Lichts von seinen Flecken waschen, Die Wahrheit, die sich sonst nie obne Schleier weist . . entkleidet uberraschen; Der Schöpfung Grundriss ubersehn' ... und Faust spricht: (Ach könnt ich doch) 'Von allem Wissensqualm entladen In deinem [des Mondes] Tau gesund mich baden' (V. $43 \mathrm{f}$.); er klagt, dass sich Natar des Schleiers nicht berauben lissot (Schröer V. 320) und strebt nach der Erkenntnis dessen, was die Welt im Innersten zusammenhalt (V. $29 \mathrm{f}$.). $Z$ wischen den hier vorsehwebenden Stollen der Musarion

*) Beides hatto Wielands Quelle, die Apomnemonenmata des Xenophon (II 1, 33) schwächer angedentet mit den

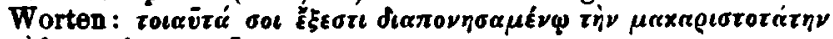

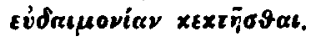


stehen die Verse: 'Nennt immer den begluckt... der.. selbst den Tod, der ihn mit Lorbeern schmückt, Wie eine Brant an seinen Busen drückt.' Indem Goethe den Vergleich zum zweiten selbständigen Moment erhebt, folgt er dem Banne seines Geduchtnisses in Fansts Worten (Schröer V. 1220 ff.) : ' $O$ selig der, dem er [der Tod] im Siegesglanze Die blut'gen Lorbeern am die Schlafe windet, Den er nach rasch durchrastem Tanze In eines Mådchens Armen findet.' Diese gewiss zutalligen Anklånge baben kein grösseres Gewicht, als dass auch sie die litterarischen Voraussetznngen verraten, aus denen der Faust erstehen konnte. Ans dem gleichen Grunde möchte ich anmerken, dass zum Homanculus in der Phiole Wieland ein Scherflein beigestenert haben kann; in den Goethe wohlbekannten Beiträgen zur geheimen Geschichte des menschlichen Verstandes and Herzens (1770 I 221) wünscht der Verfasser seine ans dem Tristram als 'Menschen im Keime' entlebnten Homanculos unter eine Glasglocke setzen zn können. Doch anch dieser Hinweis gewahrt keine Einsicht in die Entstehungszeit des Faust.

Fruchtbarer in diesem Betracht ist Wielands Gedicht An Psyche (T. Merkur 1776 I 12 ff.). Es gibt Zengnis, welche Teile der Fanstdichtung am Schlnsse des Jahres 1775 vorhanden gewesen sein milssen. Veranlassung nnd Inhalt des poetischen Briefes ist Wielands Aufenthalt in Stetten vom 1.-3. Januar 1776. Anch Goethe war dahin gekommen (Wielands Briefe an S. La Roche S. 180) und las mehrere Scenen ans seinem Faust vor: ' $O$ welche Gesichte ( $\mathrm{vgl}$. Faust V. 167), welche Scenen Hiess er vor unsern Augen entstehn!' ruft Wieland aus. 'Und wenn wir dachten, wir hatten's gefunden .. Wie ward' er Bo schnell uns wieder neu! Entschltipfte plotslich dem satten Blick Und kam in andrer Gestalt zurtick; ... Und jede der tausendfachen Gestalten So ungezwungen ....!' Unter den vorgetragenen Scenen mitssen einmal Fansts erster Monolog and seine Worte nach der grossen Ltucke 
gewesen sein ; das ergeben ausser Wielands oft vermerkter Anspielung anf Nostradamas die Verse: 'Wir fuhlten's mit allen unsern Sinnen Durch alle unsre Adern rinnen - vgl. Fanst V. 78. 80 ... Der alle Gute und alle Gowalt Der Menschbeit 80 in sich vereinigt - vgl. Fanst V. $249 \mathrm{ff} . .$. . Der nnzerdrtickt von ihrer Last So muchtig alle Natur umfasst - vgl. Faust V.85. 88... So tief in jedes Wesen sich grabt Und doch so innig im Gansen lebt!' - vgl. Faust V. 94 f 250 fi. Sodann mtissen auch Gretchenscenen vorgelesen worden sein und zwar wohl Gretchen am Spinnrad und im Zwinger, wie aus Wielands Worten hervorgeht: 'Wer schmelzt wie er die Lust im Schmerz? Wer kann so lieblich angsten und qualen? In stlssern Trünen zerschmelzen das Herz?' Auch die hier gewonnenen Anhaltspunkte fur den Torso des Herkules, wie Schiller das Fragment. nannte, stimmen mit Scherers Datiernngen uberein.

Im ersten Jahre des Erscheinens des Faustfragmentes wurden vier Ausgaben im gleichen Klein-Oktavformat verlegt in Leipzig, | beq Beorg Joadim Böjфen, | 1790. Zwei davon $(A B)$ bilden den $\Delta$ nfang von Boethe's | Sфriften. | Siebenter Sanb. Den Titel schmtickt ein auf Iery und Bately beztigliches Bildchen, vorausgesetzt ist ein Kupferblatt, Fausts erste Monologacene darstellend. Die beiden andern Ausgaben $(a b)$ sind betitelt: Faupt. Ein Fragment. | Bon | Epethe. | İdte Yugaabe. Diese Hanpttitel sind in allen selbstandig gedruckt. Das erste Blatt des Bogens $\mathfrak{A}$ trägt in allen die Specialtitel : Fruff. | Fin Fragment. Von den 168 Seiten sämmtlicher Drucke beginnt jede mit dem gleichen Worte. Die funf Bogen $\mathscr{Q}$ bis $\mathbb{E}$ aller vier Auvgaben sind von einem Satre abgezogen, wie die genaneste Ubereinstimmnng in allem, auch in schadhaften Lettern - ich habe acht Drucke verglichen - erweist, nur dass die Norm von $A B$ Boetbe's 23 . 7. $\mathfrak{B}$. auf diesen und den ubrigen Bogen in $a b$ weggelassen, auch durch keine neue er- 
setst ist. Von Bogen $\mathfrak{F}$ an aber teilen sich die Ausgaben in zwei Gruppen $A a$ and $B b$. Veranlassung 20 einem zweiten Drucke der folgenden Bogen war die grössere Anzabl von Druckfehlern, wăhrend auf den ersten nur funf Fehler sich finden. Ausser dem Lnterschiede in Gestalt und Stellung einzelner Schriftzeichen sind folgende Abweichungen bemerkbar: V. 1052 'wab $A a$ was $B b \mid 1110$ g'rabe $A a$ gr'abe $B b \mid 1119$ gejfininb, $A a$ gejdmint. $B b \mid 1145$ lejeni $A a$ lejen $B b \mid 1212$ Đörjaal $A a$ ఏörjal $B b \mid 1254$ 'was $A a$ was $B b \mid 1282$ jebem $A a$ jeben $B b \mid 1290$ Bargrethlein $A a$ Margrethletn $B b \mid$ 1363 jageu $A a$ jagen $B b \mid$ vor 1393 Margarethe. $A a$ Mephiftopheles. $B b \mid 1415$ 'mas $A a$ was $B b \mid 1490$ euer, $A a$ euer. $B b \mid 1494$ 'mas $A a$ mas $B b \mid 1511$ g'rab $A a$ g'rab' $B b \mid 1586$ ubernähm' (oder doch undentliches it) $A a$ Abernäbm' $B b$ | 1612 g'rab $A a$ g'rab' $B b$ | 1653 Berzweiflung $A a$ Berzweiflung $B b \mid 1801$ bethen. $A a$ betben, $B b \mid 1834-6$ schliessen den Bogen $S$ and sind wiederholt zu Anfang des Bogens $\Re A a$ feblen anf Bogen 3 - die vorhergehenden Zeilen sind zar Ausfullung des Raumes gedehnt - und beginnen den Bogen \& $B b \mid 1863$ ESejфled $A a$ Bejळled" $B b \mid 1913$ bolltomm'nes $A a$ Bollomm'nes $B b \mid 1975$ unb $A a$ uub

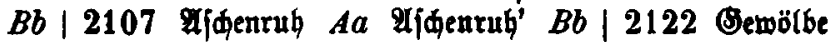
$A a$ Eemölbe, $B b$. Aus dieser Zusammenstellang ist ersichtlich, dass auf Bogen $\mathfrak{F}$ bis $\Re$ Druckfehler berichtiget und auf denselben einschliesslich \& Kleinigkeiten verundert sind, dass aber auch neue Druckfehler sich eingeschlichen haben. Im ganzen also bietet $B b$ einen verbesserten Text, welcher for die nochmals berichtigte Ausgabe in Goethes Schriften $1791 \mathrm{Bd} 4$ massgebend ward. Es ist an sich unwahrscheinlich, dass der fehlerhaftere Druck $A a$ der spatere ist, and darum nnmoglich, weil dann beim Umdrucken des Bogens 3 der Betzer die ersten Zeilen des nachsten Bogens herthergenommen haben mtlsate; allein begreiflich ist das Umgekehrte, dass beim ersten Satze nach dem Manu- 
skripte die Schlusszeilen des einen Bogens zu Beginn des andern wiederholt wurden.

Demnach hat der Verleger den Faust in den gesammelten Schriften, und zwar auf stärkerem und leichterem Papiere, $A$ und gleichzeitig als Separatausgabe $a$ herausgegeben; dies ist die erste, die Originalausgabe. Dann veranstaltete Göschen eine zweite Ausgabe in den Schriften $B$ und einzeln $b$, für welche er die ersten fünf Bogen von $A a$ benützte, die fünf letzten in äusserlich getreuester Nachahmung des ersten Satzes neu drucken liess. ${ }^{*}$ )

Diesen vier Ausgaben stehen drei zur Seite: Exemplare von $A$ wurden auch in anderer Form zu Markt gebracht; die Bandnorm ward beibehalten, der Bandtitel aber weggelassen und durch ein neues Blatt ersetzt mit der Inschrift: Fauft. | Ein Traueripiel | von | Göthe | Reipzig, | bey Georg Joadjim Göjtyen. | 1787. Ferner kennt Holland (Goethes Faust. 1882. S. VI) einen Druck mit dem Titel: Frauft. Ein Fragment. Bon Soethe.

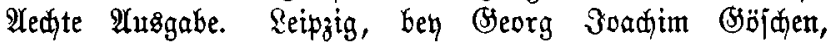
1787. Derselbe bietet ebenfalls den ersten Satz; ob $A$ oder $a$, lässt der Nachweis nicht bestimmen. Endlich ist in Wenzels 'Aus Weimar's goldenen Tagen' S. 53 eine Ausgabe verzeichnet mit der Aufschrift: $\mathfrak{F a u f t . ~ F i n ~}$ Fragment. Reipzig, Ssöjłen 1789. 8\%.168 SS. Welcher der vier ersten Ausgaben dieses neue Titelblatt vorangesetzt wurde, ist aus Wenzels Beschreibung nicht ersichtlich; eine handschriftliche Bemerkung in einem der mir vorliegenden Exemplare deutet auf $B$ oder $b$. Jedenfalls liegt den Datierungen dieser Drucke ein Irrtum (oder eine absichtliche Täuschung?) zu Grunde.

Der nachfolgende Neudruck gibt den Text von $A a$,

*) Eine Ernenerung dieser zweiten Ausgabe ist in diesen 'Tagen erschienen unter dem Titel: Goethes Faust ein Fragment in der ursprünglichen Gestalt neu herausgegeben von Holland. Freiburg i. B. und Tübingen 1882. J. C. B. Mohr. 
den Haupttitel von $a$ wieder. Verbessert sind darin ausser den oben verzeichneten Druckfehlern in V. 1145, 1282, $1290,1363,1393,1586,1653,1834$ ff. die folgenden : V. 250 meinem ans meinen | 283 frrone aus Sronen | 379

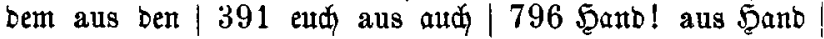
1176 marmem aus warmen 1491 foll't aus follt' vgl. 1544 fönn't aus fönnt' zwar steht V. 2016 follt 1132 fönnt und öfters wollt; aber die Anwendung des Apostrophes ist nicht gleichmässig durchgeführt und im allgemeinen zeigt sich eine Vorliebe für denselben, so dass sogar V. 1271 jah' und hier und in den Ausgaben letzter Hand 1720 hält'it gedruckt ist | 1947 oumpjem aus bumpien ।

Wie in den übrigen Drucken dieser Sammlung sollten auch hier nur die offenbaren Druckversehen beseitigt, nicht aber ein durchaus gereinigter Text hergestellt werden, weshalb die Ungleichheiten in Orthographie, Verwendung des Apostrophes, Interpunktion bewahrt wurden, zumal auch die Drucke $B b$ und 1791 neben den Berichtigungen alte Druckfehler und Inkonsequenzen aufweisen. Es blieb also unverändert: V. 85 ring's obwohl sechsmal rings steht $\mid 174 \mathrm{f}$. Rommöbiant $\mid 253$ Shrem weil in der Ausgabe 1791 auch V. 143 bu der starken Betonung wegen in $\mathfrak{D} \mathfrak{u}$ verbessert wurde. Sonst sind die Anredewörter mit kleinem Anfangsbuchstaben geschrieben ausser $\mathcal{E}_{\mathfrak{r}}$ und Sie (Sing. und Plur.) mit den entsprechenden Possessivpronomina, wonach in V. 783, $1342,1344 \mathrm{zu}$ korrigieren wäre $/ 559$ wolt' steht für molltet wie 1114 zugeriat' für zugeridutet vgl. 781 Wart 1308 Danft statt Danft' wie 1860 Bilb't - so auch in den Ausgaben letzter Hand - statt $\mathfrak{B i l}$ ' $t$ ' $\mid 1913$ vollfomm'nes statt $\mathfrak{B}_{\text {ollfonm'ne }}$ vgl. $364,822,1502$, 2045.

Die nachgenannten Stellen sollen gegen etwaigen Verdacht der Fehlerhaftigkeit von vornherein verteidigt werden : V. 187 f. blaj't . . aนtz (später 'rauz) s. Grimms D.Wb. z. B. Rauch aus der Pfeife ausblasen; vgl. 1662 
aufgeflogen | 850 und nach 927 Shorfiein 8. Adelungs Wb. / 918 reimen; der anch in den Ansgaben letzter Hand bewahrte Strichpunkt zeigt an, dass die Tiere hier keine Pause machen, sondern sugleich mit Faust und Kephistopheles fortsprechen | 2053 and 2072 schlieseon an die vorhergehenden Zeilen und Seiten ohno irgend ein Zeichen der Unterbrechnng an; auch die Ausgabe 1791 fahrt ohne Absats fort, whrend die Ausgaben letzter Hand neu anheben.

Ferner sind einjge in $A a$ undentlich ansgepragten Schriftzeichen zu vermerken, welche bei der Mehrzahl der verglichenen Abztige erkennbar, in einem oder dem andern Exemplar aber unklar oder verschwunden waren; so nach V. 976 das zweite $n$ in unanftäbige / 1169 der Punkt nach träujeln | nach 1207 der Punkt nach ju | 1236 die Bindestriche $z$ wischen Bäter = Eaale $\mid 1430$ die Trennungsstriche $\mathrm{zwischen}$ Iries ben ।

Die Scenen, welche alle mit Ausnahme der zweiten, eingangs fragmentarischen eine Zeit oder Ort bestimmende Uberschrift tragen, sind im Originale in verschiedenen Abstanden aneinander gereiht, ohne dass daraus der Schlnss anf Zusammengehörigkeit der nkher geruckten sich zieheu liesse. Mit den Scenen: Faust und Mephistopheles, Auerbachs Keller, Hexenkuche, Gretchens Stube, Wald und Höhle, Dom beginnt je eine neue Seite, an deren Kopf (ausser vor der Domscene) Raum frei gelasseu ist; die tubrigen Scenen schliessen sich eng an einsnder an.

Die Verszählung endlich stimmt mit der von Schröer eingefthrten und wohl allgemein anerkannten Ordnung therein; so auch darin, dass V. 2029 ' Unb iq, ber Oott= verbaßjte, hatte nidt genug,' wegen der daranf folgenden Reimbander in zwei Verse geteilt ist, also die Worte '\$atte niфt genug,' einen eigenen V. 2030 bilden. Nur in dem einen Falle weicht die hier angezeigte Verszahlung von der Schröerschen ab, dass sie auch V. 1643 dem gewiss richtigen Grundsatze Schroers gemllss, die gerade in der ersten Ausgabe sorgfältige Anordnung Goethes 
2u wahren, nicht mit 1644 verbindet; die reimlosen Verse beginnen 1642, (Er liekt mid! ist als selbstandiger Vers zn zahlen. Um das Nachschlagen zu erleichtern, ist im nachstehenden Texte der an den uusseren Rundern angegebenen dorchlaufenden Zählung des Fragmentes die Schroersche Zahlung an den inneren Randern in kleinerer Schrift beigefugt und oben in den Ecken der Seiten die Zublung v. Loepers mit L. angemerkt.

Wifzbarg, Anfang Febraar 1882.

\section{Bernhard Senffert.}

Berichtigungev.

V. 912 lies halt' | 1954 gönnen. | 
